\title{
EVALUATION OF VARIOUS WIENER INTEGRALS BY USE OF CERTAIN STURM-LIOUVILLE DIFFERENTIAL EQUATIONS
}

\author{
R. H. CAMERON AND W. T. MARTIN
}

Introduction. Wiener [1] $]^{1}$ has introduced a measure in the space $C$ consisting of all real-valued functions $x(t)$ continuous on $0 \leqq t \leqq 1$ and vanishing at $t=0$. He first defines the measure of a quasi-interval-a quasi-interval is the set of all functions of $C$ which satisfy relations of the form

$$
a_{i}<x\left(t_{j}\right)<b_{i}, \quad j=1, \cdots, n,
$$

where $n$ is a positive integer, $a_{j}$ and $b_{j}$ are real numbers, $-\infty \leqq a_{j}<b_{j}$ $\leqq \infty$, and $t_{1}, \cdots, t_{n}$ are $n$ ordered points on $0<t \leqq 1,0<t_{1}<\cdots$ $<t_{n} \leqq 1$. The Wiener measure of this quasi-interval is defined to be

$$
\begin{aligned}
& \frac{1}{\left(\pi^{n} t_{1}\left(t_{2}-t_{1}\right) \cdots\left(t_{n}-t_{n-1}\right)\right)^{1 / 2}} \int_{a 1}^{b_{1}} \cdots \\
& \cdot \int_{a_{n}}^{b_{n}} \exp \left(-\frac{s_{1}^{2}}{t_{1}}-\frac{\left(s_{2}-s_{1}\right)^{2}}{t_{2}-t_{1}}-\cdots-\frac{\left(s_{n}-s_{n-1}\right)^{2}}{t_{n}-t_{n-1}}\right) d s_{1} \cdots d s_{n},
\end{aligned}
$$

the multiplier before the integrals having been so chosen as to make the measure of the entire space $C$ equal to unity. With this definition of measure for quasi-intervals, the Wiener measure on $C$ can be defined in a manner entirely similar to that used for ordinary Lebesgue measure, and a theory of (Wiener) integration can be defined over $C$, the integral of a functional $F[x]$ being a number. We write

$$
\int_{S}^{W} F[x] d d_{w} x
$$

to denote the Wiener integral of a functional $F[x]$ over a subset $S$ of the space $C$.

In several papers the authors have recently considered various aspects of the theory; in the present paper we shall evaluate several Wiener integrals. In particular, we shall evaluate integrals of the form

$$
\int_{C}^{W} \exp \left(\lambda \int_{0}^{1} p(t)[x(t)+g(t)]^{2} d t\right) d_{w} x
$$

Presented to the Society, August 13, 1944; received by the editors June 5, 1944.

${ }^{1}$ Numbers in brackets refer to the bibliography at the end of the paper. 
or, more generally,

$$
\int_{C}^{W} \exp \left(\lambda \int_{0}^{1} p(t) x^{2}(t) d t\right) F\left[\int_{0}^{1} g(t) x(t) d t\right] d_{w} x
$$

where $p(t)$ is a positive-valued continuous function on $0 \leqq t \leqq 1, g(t)$ is real and of class $L_{2}$ on $0 \leqq t \leqq 1$, and $\lambda$ is real and less than a suitable positive constant $\lambda_{0}$ which depends only upon the function $p(t)$. In (0.3) the function $F(u)$ will be a fairly general Lebesgue measurable function on $-\infty<u<\infty$.

In a recent paper [2] we have shown that

$$
\int_{C}^{W} \exp \left(\lambda \int_{0}^{1} x^{2}(t) d t\right) d_{w} x=\left(\sec \lambda^{1 / 2}\right)^{1 / 2}, \quad 0 \leqq \lambda<\pi^{2} / 4 .
$$

This integral was evaluated by making use of a suitable linear transformation of $C$ into itself; namely, the transformation

$$
y(t)=x(t)+\lambda^{1 / 2} \int_{0}^{t} \tan \lambda^{1 / 2}(s-1) \cdot x(s) d s .
$$

Under this transformation we found that

$$
\begin{aligned}
1=\int_{C}^{W} 1 \cdot d_{w} y=\left(\cos \lambda^{1 / 2}\right)^{1 / 2} \int_{C}^{W} \exp \left(\lambda \int_{0}^{1} x^{2}(t) d t\right) d_{w} x \\
0 \leqq \lambda<\pi^{2} / 4
\end{aligned}
$$

and from this (0.4) was obtained. The evaluation of integrals of the form

$$
\int_{C}^{W} \exp \left(\lambda \int_{0}^{1} p(t) x^{2}(t) d t\right) d_{w} x, \quad-\infty<\lambda<\lambda_{0},
$$

can be carried out in a similar fashion using suitable linear transformations; the particular linear transformation to be used in each case will be determined by a consideration of the second order differential equation

$$
f^{\prime \prime}(t)+\lambda p(t) f(t)=0
$$

and the value $\lambda_{0}$ will be the least characteristic value of the equation (0.8) corresponding to the boundary values $f(0)=f^{\prime}(1)=0$.

The evaluation of integrals of the form (0.3) will use the same range of ideas, together with certain results recently obtained. Integrals of the form (0.2) are special cases of (0.3).

The evaluation of $(0.7)$ is given by the following theorem. 
THEOREM 1. Let $p(t)$ be continuous and positive $(>0)$ on $0 \leqq t \leqq 1$ and let $\lambda_{0}$ be the least characteristic value of (0.8) subject to the boundary conditions

$$
f(0)=f^{\prime}(1)=0 .
$$

Then if $-\infty<\lambda<\lambda_{0}$ and $f_{\lambda}(t)$ is any non-trivial solution of $(0.8)$ satisfying $f_{\lambda}^{\prime}(1)=0$, we have

$$
\int_{C}^{W} \exp \left(\lambda \int_{0}^{1} p(t) x^{2}(t) d t\right) d_{w} x=\left(\frac{f_{\lambda}(1)}{f_{\lambda}(0)}\right)^{1 / 2}
$$

Consequently, if $\lambda<\lambda_{0}$ and $f^{(1)}(t)$ and $f^{(2)}(t)$ are any two linearly independent solutions of $(0.8)$, we have

$$
\begin{aligned}
\int_{C}^{W} \exp \left(\lambda \int_{0}^{1} p(t) x^{2}(t) d t\right. & d_{w} x \\
& =\left(\frac{f^{(2)^{\prime}}(a) f^{(1)}(a)-f^{(1)^{\prime}}(a) f^{(2)}(a)}{f^{(2)^{\prime}}(1) f^{(1)}(0)-f^{(1) '}(1) f^{(2)}(0)}\right)^{1 / 2}
\end{aligned}
$$

where $a$ is any convenient point in $0 \leqq a \leqq 1$.

Remark 1. By the hypotheses of Theorem $1, \lambda_{0}$ must exist as a positive number.

Remark 2. For every value of $\lambda$ there always exist (non-trivial) solutions of $(0.8)$ satisfying $f_{\lambda}^{\prime}(1)=0$.

Remark 3. The numerator inside the square root sign in (0.11) is a Wronskian whose value is actually independent of $a, 0 \leqq a \leqq 1$.

Theorem 1 is a special case of Theorem 1a, which we shall prove in $\S 1$.

THEOREM 1a. Let $p(t)$ be continuous and positive on $0 \leqq t \leqq 1$, and let $\lambda_{0}$ be the least characteristic value of the differential equation (0.8) subject to the boundary conditions (0.9). Then if $F(x)$ is any Wiener measurable functional, if $\lambda<\lambda_{0}$, and if $f_{\lambda}(t)$ is any non-trivial solution of (0.8) satisfying $f_{\lambda}^{\prime}(1)=0$, we have

$$
\begin{aligned}
& \int_{C}^{W} F(x) \exp \left(\lambda \int_{0}^{1} p(t) x^{2}(t) d t\right) d_{w} x \\
& \quad=\left(\frac{f_{\lambda}(1)}{f_{\lambda}(0)}\right)^{1 / 2} \int_{C}^{W} F\left[y(\cdot)+f_{\lambda}(\cdot) \int_{0}^{(\cdot)} \frac{f_{\lambda}^{\prime}(s)}{\left[f_{\lambda}(s)\right]^{2}} y(s) d s\right] d_{w} y,
\end{aligned}
$$

where the existence of either Wiener integral implies the existence of the other.

Remark 4 . The radical on the right-hand side of $(0.12)$ can be re- 
placed by the radical on the right-hand side of $(0.11)$.

By using Theorem 1a and certain results proved recently we shall obtained in $\$ 2$ the following result.

THEOREM 2. Let $g(t)$ be real and of class $L_{2}$ on $0 \leqq t \leqq 1$, let $p(t)$ be $a$ positive-valued continuous function on $0 \leqq t \leqq 1$, let $\lambda_{0}$ be the least characteristic value of the system $(0.8),(0.9)$ and let $f_{\lambda}(t)$ be any non-trivial solution of $(0.8)$ satisfying $f_{\lambda}^{\prime}(1)=0$. Then

$$
\begin{gathered}
\int_{C}^{W} \exp \left(\lambda \int_{0}^{1} p(t) x^{2}(t) d t\right) F\left[\int_{0}^{1} g(t) x(t) d t\right] d_{w} x \\
=\left(\frac{f_{\lambda}(1)}{\pi \cdot f_{\lambda}(0)}\right)^{1 / 2} \int_{-\infty}^{\infty} F(b u) e^{-u^{2}} d u
\end{gathered}
$$

where

$$
b^{2}=\int_{0}^{1}\left[\frac{1}{f_{\lambda}(t)} \int_{1}^{t} g(s) f_{\lambda}(s) d s\right]^{2} d t,
$$

the result holding and the Wiener integral being convergent whenever

$$
F(b u) e^{-u^{2}} \in L_{1}(-\infty, \infty) .
$$

In the final sections we consider a few choices of $p(t)$ for which the differential equation $(0.8)$ has solutions in terms of well known functions. Various other choices may occur to the reader.

1. Proofs of Theorems 1a and 1. We shall need certain well known properties of the differential equation (0.8). First, by classical SturmLiouville theory it is known that there is a least characteristic value $\lambda_{0}$ of the system $[(0.8),(0.9)]$, it is known that $\lambda_{0}$ is positive, and it is known that the solution $f(t)$ of the system $[(0.8),(0.9)]$ with $\lambda=\lambda_{0}$ is nonvanishing in $0<t \leqq 1$. These facts are all given for example in [3]. We need one other (known) property; namely, the following:

RESULT 1. Every non-trivial solution $f_{\lambda}(t)$ of $(0.8)$ corresponding to any real value of $\lambda<\lambda_{0}$ and satisfying the single boundary condition

$$
f_{\lambda}^{\prime}(1)=0
$$

is nonvanishing in $0 \leqq t \leqq 1$.

For the sake of completeness we shall give a classical proof of this result. For the proof we use the identity

$$
\left[f^{\prime} f_{\lambda}-f f_{\lambda}^{\prime}\right]_{t}^{1}=\left(\lambda-\lambda_{0}\right) \int_{t}^{1} p(s) f(s) f_{\lambda}(s) d s,
$$


where $0 \leqq t \leqq 1, \lambda<\lambda_{0}, f_{\lambda}(t)$ is a non-trivial solution of (0.8) satisfying (1.1), and $f(t)$ is a solution of the system $[(0.8),(0.9)]$ with $\lambda=\lambda_{0}$. Now $f_{\lambda}(t)$ cannot vanish at $t=0$ since $\lambda_{0}$ is the least characteristic value of the system $[(0.8),(0.9)]$. Also it cannot vanish at $t=1$ for if it did it would be identically zero. If it vanishes at all in $0 \leqq t \leqq 1$ its zeros are isolated and we shall denote by $t_{0}$ its greatest zero in $(0,1)$. With this value of $t_{0}(<1)$ relation $(1.2)$ becomes

$$
f\left(t_{0}\right) f_{\lambda}^{\prime}\left(t_{0}\right)=\left(\lambda-\lambda_{0}\right) \int_{t_{0}}^{1} p(s) f(s) f_{\lambda}(s) d s .
$$

Without losing generality we assume

$$
\begin{array}{rlrl}
f_{\lambda}(t) & >0, & & t_{0}<t \leqq 1, \\
f(t) & >0, & 0 & 0 t \leqq 1 .
\end{array}
$$

Then (1.3) yields the result that $f_{\lambda}^{\prime}\left(t_{0}\right)$ is negative. Since $f_{\lambda}\left(t_{0}\right)$ is assumed zero this contradicts (1.4). Hence there is no greatest zero of $f_{\lambda}(t)$ in $0<t \leqq 1$. This yields Result 1 .

We now proceed to the proof of Theorem 1a. For this purpose we consider the linear transformation

$$
y(t)=x(t)-\int_{0}^{t} \frac{f_{\lambda}^{\prime}(s)}{f_{\lambda}(s)} x(s) d s,
$$

where $\lambda$ is fixed, $-\infty<\lambda<\lambda_{0}$, and $f_{\lambda}(t)$ is any non-trivial solution of (0.8) satisfying (1.1). (By Result 1 , the denominator of the integral in (1.6) does not vanish.) Obviously the transformation (1.6) takes the space $C$ into a part of $C$. We shall show that it actually takes $C$ into the whole of $C$ in a 1-1 manner. First

so that

$$
\begin{aligned}
\frac{f_{\lambda}^{\prime}(t)}{\left[f_{\lambda}(t)\right]^{2}} y(t) & =\frac{f_{\lambda}^{\prime}(t)}{\left[f_{\lambda}(t)\right]^{2}} x(t)-\frac{f_{\lambda}^{\prime}(t)}{\left[f_{\lambda}(t)\right]^{2}} \int_{0}^{t} \frac{f_{\lambda}^{\prime}(s)}{f_{\lambda}(s)} x(s) d s \\
& =\frac{d}{d t}\left[\frac{1}{f_{\lambda}(t)} \int_{0}^{t} \frac{f_{\lambda}^{\prime}(s)}{f_{\lambda}(s)} x(s) d s\right]
\end{aligned}
$$

$$
\int_{0}^{t} \frac{f_{\lambda}(s)}{\left[f_{\lambda}(s)\right]^{2}} y(s) d s=\frac{1}{f_{\lambda}(t)} \int_{0}^{t} \frac{f_{\lambda}^{\prime}(s)}{f_{\lambda}(s)} x(s) d s .
$$

Multiplying (1.8) by $f_{\lambda}(t)$ and adding to (1.6), we obtain

$$
y(t)+f_{\lambda}(t) \int_{0}^{t} \frac{f_{\lambda}^{\prime}(s)}{\left[f_{\lambda}(s)\right]^{2}} y(s) d s=x(t) .
$$


Thus to every function $x(\cdot)$ of $C$ there corresponds a function $y(\cdot)$ of $C$ by (1.6), and this $y(\cdot)$ satisfies the relation (1.9). Conversely, it is easily seen that if $y(\cdot)$ is any function of $C$ and if $x(\cdot)$ is defined by (1.9), then $x(\cdot)$ and $y(\cdot)$ again satisfy (1.6). Hence (1.6) is a 1-1 transformation of $C$ onto itself. This could also be seen from the fact that (1.6) is a Volterra transformation. We shall consider it, however, as a Fredholm transformation whose kernel vanishes when $t<s$, and calculate the Fredholm determinant using the half (or arithmetic mean) value along the diagonal :

$$
\begin{aligned}
D & =\exp \left(\frac{1}{2} \int_{0}^{1}\left\{-\frac{f_{\lambda}^{\prime}(s)}{f_{\lambda}(s)}\right\} d s\right) \\
& =\exp \left(-\frac{1}{2}\left[\log f_{\lambda}(1)-\log f_{\lambda}(0)\right]\right)=\left(\frac{f_{\lambda}(0)}{f_{\lambda}(1)}\right)^{1 / 2} .
\end{aligned}
$$

In Theorem I of a recent paper [2] we have considered the behavior of Wiener integrals under Fredholm and Volterra transformations,

where

$$
y(t)=x(t)+\int_{0}^{1} K(t, s) x(s) d s,
$$

$$
K(t, s)= \begin{cases}K^{(1)}(t, s) & \text { when } 0 \leqq t<s, 0<s \leqq 1, \\ K^{(2)}(t, s) & \text { when } s<t \leqq 1,0 \leqq s<1, \\ K^{(1)}(s, s) / 2+K^{(2)}(s, s) / 2 & \text { when } t=s, \quad 0 \leqq s \leqq 1 .\end{cases}
$$

We quote for reference the special case of this theorem in which $K^{(1)}(t, s) \equiv 0$, so that the transformation is a pure Volterra transformation:

Theorem A. Let $K(t, s)$ be continuous on the closed triangle $[0 \leqq s \leqq t$, $0 \leqq t \leqq 1]$, and let it satisfy the following five conditions:

A. For almost all $s, K(t, s)$ is absolutely continuous in $t, s \leqq t \leqq 1$.

B. For almost all $s, \partial K(t, s) / \partial t$ is essentially of bounded variation, $s \leqq t \leqq 1$. More precisely, there exists a measurable function $\tilde{K}(t, s)$ which is of bounded variation in $t$ for each $s$ and which for almost all $t, s$ in the triangle $[s \leqq t \leqq 1,0 \leqq s \leqq 1]$ is equal to $\partial K(t, s) / \partial t$. (In the remaining statements, the hypotheses will be considered satisfied when $\partial K / \partial t$ is replaced by such a $\tilde{K}$.)

C. $\int_{0}^{1} \sup _{s} \unlhd_{t} \leqq_{1}|\partial K(t, s) / \partial t| d s<\infty$.

D. $\int_{0}^{1} \operatorname{var}_{s} \leqq_{t} \leqq_{1}[\partial K(t, s) / \partial t] d s<\infty$.

E. $K(s, s)$ is of bounded variation $0 \leqq s \leqq 1$.

Let $S$ be a Wiener measurable subset of $C$, and let TS be the image of $S$ under the transformation 
T:

$$
y(t)=x(t)+\int_{0}^{t} K(t, s) x(s) d s
$$

Then if $\mathcal{F}(y)$ is any Wiener measurable functional for which either member of the following equation exists, the other member exists and the equality holds:

$$
\int_{T S}^{W} \mathcal{F}[y] d_{w} y=\mathcal{D} \cdot \int_{S}^{W} \mathcal{F}\left[x(\cdot)+\int_{0}^{(\cdot)} K(\cdot, s) x(s) d s\right] \exp (-\Phi[x]) d_{w} x,
$$

where

$$
\begin{aligned}
\Phi[x]= & \int_{0}^{1}\left[\frac{d}{d t} \int_{0}^{t} K(t, s) x(s) d s\right]^{2} d t \\
& +2 \int_{0}^{1}\left[\int_{0}^{t} \frac{\partial}{\partial t} K(t, s) x(s) d s\right] d x(t) \\
& +\int_{0}^{1} K(t, t) d\left\{[x(t)]^{2}\right\}
\end{aligned}
$$

and $\mathcal{D}$ is the Fredholm determinant of the transformation, using the half (or arithmetic mean) value of the kernel on the diagonal:

$$
\begin{aligned}
\mathcal{D} & =\sum_{n=0}^{\infty} \frac{1}{n !} \int_{0}^{1} \cdots \int_{0}^{1} \frac{K\left(s_{1}, s_{1}\right)}{2} \frac{K\left(s_{2}, s_{2}\right)}{2} \cdots \frac{K\left(s_{n}, s_{n}\right)}{2} d s_{1} d s_{2} \cdots d s_{n} \\
& =\exp \left(\frac{1}{2} \int_{0}^{1} K(s, s) d s\right) .
\end{aligned}
$$

It is clear that the linear transformation (1.6) for $\lambda<\lambda_{0}$ satisfies the hypotheses of Theorem $\mathrm{A}$, and hence by that theorem with $\mathcal{F}[y]=F[x]=F\left[T^{-1}(y)\right]$, we have

$$
\begin{aligned}
\int_{C}^{W} F[y(\cdot)+ & \left.f_{\lambda}(\cdot) \int_{0}^{(\cdot)} \frac{f_{\lambda}^{\prime}(s)}{\left\{f_{\lambda}(s)\right\}^{2}} y(s) d s\right] d_{w} y \\
& =D \int_{C}^{W} F[x] \exp \left\{-\int_{0}^{1}\left[\frac{f_{\lambda}^{\prime}(s)}{f_{\lambda}(s)} x(s)\right]^{2} d s\right. \\
& \left.+\int_{0}^{1} \frac{f_{\lambda}^{\prime}(s)}{f_{\lambda}(s)} d[x(s)]^{2}\right\} d_{w} x
\end{aligned}
$$

where the existence of either side implies the existence of the other.

On integrating by parts, simplifying, using the boundary condition (1.1) and the differential equation (0.8), we obtain that (1.11) is equal to 


$$
\begin{aligned}
& D \int_{C}^{W} F[x] \exp \left\{-\int_{0}^{1}\left[\frac{f_{\lambda}^{\prime}(s)}{f_{\lambda}(s)} x(s)\right]^{2} d s-\int_{0}^{1} x^{2}(s) d\left[\frac{f_{\lambda}^{\prime}(s)}{f_{\lambda}(s)}\right]\right\} d_{w} x \\
& \quad=D \int_{C}^{W} F[x] \exp \left\{-\int_{0}^{1} \frac{\left[f_{\lambda}^{\prime}(s)\right]^{2}+f_{\lambda}(s) f_{\lambda}^{\prime \prime}(s)-\left[f_{\lambda}^{\prime}(s)\right]^{2}}{\left[f_{\lambda}(s)\right]^{2}} x^{2}(s) d s\right\} d_{w} x \\
& \quad=D \int_{C}^{W} F[x] \exp \left\{-\int_{0}^{1} \frac{f_{\lambda}^{\prime \prime}(s)}{f_{\lambda}(s)} x^{2}(s) d s\right\} d_{w} x \\
& =D \int_{C}^{W} F[x] \exp \left(\lambda \int_{0}^{1} p(s) x^{2}(s) d s\right) d_{w} x .
\end{aligned}
$$

This, together with (1.10), yields Theorem 1a, including the existence of the integrals. Relation (0.10) 1 is obtained by specializing the function $F$ in Theorem 1 to be identically unity, $F(u) \equiv 1$.

Now take $f_{\lambda}(t)=f^{(2)^{\prime}}(1) f^{(1)}(t)-f^{(1)^{\prime}}(1) f^{(2)}(t)$ where $f^{(1)}(t)$ and $f^{(2)}(t)$ are any two linearly independent solutions of $(0.8)$. This function obviously satisfies the condition $f_{\lambda}^{\prime}(1)=0$ and yields $(0.11)$ with $a=1$. But since the differential equation $(0.8)$ has no term in $f^{\prime}(t)$, its Wronskian is constant, and hence we may use any convenient point $a$ instead of $a=1$. This yields Theorem 1 .

2. Proof of Theorem 2. In a recent paper [4, Lemma 3] we gave a new proof of a special case of a theorem of Paley and Wiener [5]. We proved : Let

Let $m(t)$ be real and of bounded variation on $0 \leqq t \leqq 1$, and let $m(1)=0$.

$$
b^{2}=\int_{0}^{1} m^{2}(t) d t
$$

and let $F(u)$ be a (real or complex-valued) measurable functional defined on $-\infty<u<\infty$. Then a necessary and sufficient condition that

$$
F\left[\int_{0}^{1} x(t) d m(t)\right]
$$

be a Wiener summable functional of $x(\cdot)$ over $C$ is that

$$
e^{-u^{2} F(b u)}
$$

be of class $L_{1}$ on $-\infty<u<\infty$. Moreover, if this condition is satisfied,

2 Added in proof. In a letter to one of the authors, Professor J. L. Doob recently pointed out that this result, for an arbitrary function $m(t)$ of $L_{2}$, follows at once from probability considerations. The probability argument uses the known fact that $\int_{0}^{1} m(t) d x(t)$ is a Gaussian variable with mean zero and dispersion $b^{2} / 2$. 


$$
\int_{C}^{W} F\left[\int_{0}^{1} x(t) d m(t)\right] d_{w} x=\frac{1}{\pi^{1 / 2}} \int_{-\infty}^{\infty} e^{-u^{2} F(b u) d u . ~}
$$

On integrating by parts and using the fact that $m(1)=0$ we find from (2.4) that

$$
\int_{C}^{W} F\left[\int_{0}^{1} m(t) d x(t)\right] d_{w} x=\frac{1}{\pi^{1 / 2}} \int_{-\infty}^{\infty} e^{-u^{2}} F(b u) d u
$$

holds under the same conditions.

We pass now to the proof of Theorem 2 itself. We first consider the integral which occurs in the left-hand member of (0.13); namely,

$$
\int_{C}^{W} \exp \left(\lambda \int_{0}^{1} p(t) x^{2}(t) d t\right) F\left[\int_{0}^{1} g(t) x(t) d t\right] d_{w} x,
$$

where $g(t)$ and $p(t)$ are real, $g(t)$ of class $L_{2}(0,1)$ and $p(t)$ positive and continuous, $0 \leqq t \leqq 1$. On applying relation $(0.12)$ of Theorem 1 a for $\lambda<\lambda_{0}$ we find that (2.6) is equal to

$$
\begin{aligned}
\left(\frac{f_{\lambda}(1)}{f_{\lambda}(0)}\right)^{1 / 2} \int_{C}^{W} F & {\left[\int_{0}^{1} g(t) y(t) d t\right.} \\
& \left.+\int_{0}^{1} g(t) f_{\lambda}(t)\left\{\int_{0}^{t} \frac{f_{\lambda}^{\prime}(s)}{\left\{f_{\lambda}(s)\right\}^{2}} y(s) d s\right\} d t\right] d_{w} y,
\end{aligned}
$$

the equality holding for every measurable function $F$ for which either (2.6) or (2.7) exists. We now consider the argument of $F$ in (2.7) and integrate twice by parts; we obtain

$$
\begin{aligned}
& \int_{0}^{1} g(t) y(t) d t+\int_{0}^{1} g(t) f_{\lambda}(t)\left\{\int_{0}^{t} \frac{f_{\lambda}^{\prime}(s)}{\left\{f_{\lambda}(s)\right\}^{2}} y(s) d s\right\} d t \\
& =\int_{0}^{1} g(t) y(t) d t \\
& \quad+\int_{0}^{1} g(t) f_{\lambda}(t)\left\{-\left[\frac{y(s)}{f_{\lambda}(s)}\right]_{0}^{t}+\int_{0}^{t} \frac{d y(s)}{f_{\lambda}(s)}\right\} d t \\
& =\int_{0}^{1} g(t) y(t) d t-\int_{0}^{1} g(t) f_{\lambda}(t) \frac{y(t)}{f_{\lambda}(t)} d t \\
& \quad+\int_{0}^{1} g(t) f_{\lambda}(t)\left\{\int_{0}^{t} \frac{d y(s)}{f_{\lambda}(s)}\right\} d t \\
& =\int_{0}^{1} g(t) f_{\lambda}(t)\left\{\int_{0}^{t} \frac{d y(s)}{f_{\lambda}(s)}\right\}^{d t} \\
& =\left[G(t, \lambda) \int_{0}^{t} \frac{d y(s)}{f_{\lambda}(s)}\right]_{0}^{1}-\int_{0}^{1} \frac{G(t, \lambda)}{f_{\lambda}(t)} d y(t),
\end{aligned}
$$


where

$$
G(t, \lambda)=\int_{1}^{t} g(s) f_{\lambda}(s) d s
$$

Now $G(t, \lambda)$ vanishes at $t=1$, and $\int_{0}^{t} d y(s) / f_{\lambda}(s)$ vanishes at $t=0$. Hence (2.8) becomes

$$
-\int_{0}^{1} \frac{G(t, \lambda)}{f_{\lambda}(t)} d y(t)
$$

and hence the quantities (2.6) and (2.7) are equal to

$$
\left(\frac{f_{\lambda}(1)}{f_{\lambda}(0)}\right)^{1 / 2} \int_{C}^{W} F\left[-\int_{0}^{1} \frac{G(t, \lambda)}{f_{\lambda}(t)} d y(t)\right] d_{w} y
$$

provided any one of the (Wiener) integrals in (2.6), (2.7), or (2.11) exists. Now the function $G(t, \lambda) / f_{\lambda}(t)$ is of bounded variation on $0 \leqq t \leqq 1$, and it vanishes at $t=1$. Hence the result (2.5) applies to (2.11), yielding the result that the integral in (2.11) is equal to

$$
\frac{1}{\pi^{1 / 2}} \int_{-\infty}^{\infty} F(b u) \cdot e^{-u^{2}} d u
$$

where

$$
b^{2}=\int_{0}^{1}\left[\frac{G(t, \lambda)}{f_{\lambda}(t)}\right]^{2} d t=\int_{0}^{1}\left[\frac{1}{f_{\lambda}(t)} \int_{1}^{t} g(s) f_{\lambda}(s) d s\right]^{2} d t .
$$

This yields Theorem 2, including the summability of the integral.

3. A corollary to Theorem 2. On specializing the function $F(u)$ of Theorem 2 to be

$$
F(u)=e^{2 \lambda u}
$$

and replacing $g(t)$ by $p(t) g(t)$, we obtain from Theorem 2 the relation

$$
\begin{aligned}
\int_{C}^{W} \exp \left(\lambda \int_{0}^{1}\left[p(t) x^{2}(t)+2 p(t) g(t) x(t)\right] d t\right) d_{w} x \\
=\left(\frac{f_{\lambda}(1)}{f_{\lambda}(0)}\right)^{1 / 2} \frac{1}{\pi^{1 / 2}} \int_{-\infty}^{\infty} e^{2 \lambda \beta u} e^{-u^{2}} d u=\left(\frac{f_{\lambda}(1)}{f_{\lambda}(0)}\right)^{1 / 2} e^{\lambda^{2} \beta^{2}}
\end{aligned}
$$

where

$$
\beta^{2}=\int_{0}^{1}\left[\frac{1}{f_{\lambda}(t)} \int_{1}^{t} p(s) g(s) f_{\lambda}(s) d s\right]^{2} d t
$$


On multiplying the first and third members of (3.2) by $\exp \left(\lambda \int_{0}^{1} p(t) g^{2}(t) d t\right)$ we obtain the following corollary.

Corollary 1.

$$
\begin{aligned}
\int_{C}^{W} \exp \left(\lambda \int_{0}^{1} p(t)\right. & \left.\{x(t)+g(t)\}^{2} d t\right) d_{w} x \\
= & \left(\frac{f_{\lambda}(1)}{f_{\lambda}(0)}\right)^{1 / 2} \exp \left(\lambda^{2} \beta^{2}+\lambda \int_{0}^{1} p(t) g^{2}(t) d t\right),
\end{aligned}
$$

the equality holding for $\lambda<\lambda_{0}$ and for every real-valued function $g(t)$ belonging to $L_{2}$ on $0<t<1$. The constant $\beta$ in (3.4) is defined in (3.3).

This evaluates the expression $(0.2)$ mentioned in the introduction. In the remaining sections we consider briefly a few examples.

4. Example 1, $p(t) \equiv 1$. For $p(t) \equiv 1$, the differential equation $(0.8)$ becomes

$$
f^{\prime \prime}(t)+\lambda f(t)=0
$$

and the least characteristic value is

$$
\lambda_{0}=\pi^{2} / 4 \text {. }
$$

A non-trivial solution of (4.1) satisfying $f_{\lambda}^{\prime}(1)=0$ is

$$
f_{\lambda}(t)=\cos \lambda^{1 / 2}(t-1) .
$$

Relation (0.10) of Theorem 1 yields

$$
\int_{C}^{W} \exp \left(\lambda \int_{0}^{1} x^{2}(t) d t\right)=\left(\frac{1}{\cos \lambda^{1 / 2}}\right)^{1 / 2}, \quad-\infty<\lambda<\pi^{2} / 4 ;
$$

which agrees with result (0.4) stated in the introduction.

For use elsewhere we state explicitly Corollary 1 of $\S 4$ for $p(t) \equiv 1$.

Corollary 2. Let $g(t)$ be a real-valued function belonging to $L_{2}$ on $0<t<1$, and let $-\infty<\lambda<\pi^{2} / 4$. Then

$$
\begin{gathered}
\int_{C}^{W} \exp \left(\lambda \int_{0}^{1}[x(t)+g(t)]^{2} d t\right) d_{w} x \\
=\left(\sec \lambda^{1 / 2}\right)^{1 / 2} \exp \left(\lambda^{2} \int_{0}^{1}\left[G_{0}(t, \lambda) \sec \lambda^{1 / 2}(t-1)\right]^{2} d t\right) \\
\cdot \exp \left(\lambda \int_{0}^{1} g^{2}(t) d t\right)
\end{gathered}
$$


where

$$
G_{0}(t, \lambda)=\int_{1}^{t} g(s) \cos \lambda^{1 / 2}(s-1) d s .
$$

5. Example 2, $p(t)=(t+\alpha)^{-2}, 0<\alpha<\infty$. With this value of $p(t)$ the differential equation $(0.8)$ becomes

$$
f^{\prime \prime}(t)+\left(\lambda /(t+\alpha)^{2}\right) f(t)=0
$$

and if we write

$$
\lambda=1 / 4-\mu^{2},
$$$$
0<\mu<\infty
$$

then two linearly independent solutions are

$$
(t+\alpha)^{\mu+1 / 2}
$$

and

$$
(t+\alpha)^{-\mu+1 / 2} \text {. }
$$

Also it is easily seen that $\lambda_{0} \geqq 1 / 4$ since no linear combination of (5.3) and (5.4) satisfies the boundary condition

$$
f(0)=f^{\prime}(1)=0 .
$$

Hence relation ( 0.11 ) of Theorem 1 yields

$$
\begin{aligned}
\int_{C}^{W} \exp & \left(\left(1 / 4-\mu^{2}\right) \int_{0}^{1} \frac{x^{2}(t)}{(t+\alpha)^{2}} d t\right) d_{w} x \\
= & \left(\frac{2 \mu \alpha^{\mu-1 / 2}(1+\alpha)^{\mu+1 / 2}}{(\mu-1 / 2) \alpha^{2 \mu}+(\mu+1 / 2)(1+\alpha)^{2 \mu}}\right)^{1 / 2}, \\
& 0<\mu<\infty, 0<\alpha<\infty .
\end{aligned}
$$

6. Example 3, $p(t)=e^{\alpha t}, \alpha$ real, $\alpha \neq 0$. With this choice of $p(t)$ the differential equation $(0.8)$ becomes

$$
f^{\prime \prime}(t)+\lambda e^{\alpha t} f(t)=0 \text {. }
$$

We solve (6.1) by use of a series with undetermined coefficients-let

Then (formally)

$$
f(t)=\sum_{n=0}^{\infty} a_{n} e^{n \alpha t}
$$

$$
f^{\prime \prime}(t)=\sum_{n=0}^{\infty} a_{n} \cdot n^{2} \alpha^{2} e^{n \alpha t}
$$

and (6.1) leads to 


$$
\sum_{n=0}^{\infty} a_{n} n^{2} \alpha^{2} e^{n \alpha t}=-\lambda \sum_{n=0}^{\infty} a_{n} e^{(n+1) \alpha t}=-\lambda \sum_{n=1}^{\infty} a_{n-1} e^{n \alpha t} .
$$

On equating coefficients we find

or

$$
a_{n} n^{2} \alpha^{2}=-\lambda a_{n-1}, \quad n=1,2,3, \cdots,
$$

$$
a_{n}=-\lambda \frac{a_{n-1}}{n^{2} \alpha^{2}}=\frac{(-\lambda)^{n}}{(n !)^{2} \alpha^{2 n}} a_{0} .
$$

Choosing $a_{0}=1$ we find

$$
f(t)=\sum_{n=0}^{\infty}(-1)^{n} \frac{\lambda^{n}}{\alpha^{2 n}} \frac{e^{n \alpha t}}{(n !)^{2}}
$$

and it is easily seen that this is an actual solution, not just a formal one. Also the series (6.4) is the classical Bessel function of order zero,

$$
J_{0}\left(2 \lambda^{1 / 2} e^{\alpha t / 2} / \alpha\right) \text {. }
$$

The function (6.5) is a solution of (6.1) for every value of $\lambda$, $-\infty<\lambda<\infty$. We note that $J_{0}(z)$ is an even (entire) function and hence there is no difficulty in (6.5) when $\lambda$ is negative.

A second linearly independent solution of (6.1) for $\lambda$ real and not equal to 0 is given by

$$
f^{(2)}(t)=Y_{0}\left(2 \lambda^{1 / 2} e^{\alpha t / 2} / \alpha\right),
$$

where $Y_{0}(z)$ is the Bessel function of the second kind of order zero, as introduced by Weber and Schläfli. The general Bessel function of the second kind is defined by the equation

$$
Y_{n}(z)=\frac{J_{n}(z) \cos n \pi-J_{-n}(z)}{\sin n \pi}
$$

or by the limit of this expression when $n$ is an integer. See [6, pp. 370, 371.] Denoting (6.5) by $f^{(1)}(t)$ we next calculate the expression

$$
f^{(2)^{\prime}}(a) f^{(1)}(a)-f^{(1)^{\prime}}(a) f^{(2)}(a),
$$

which occurs in relation ( 0.11$)$ of Theorem 1 . It is easily seen that (6.8) is equal to

$$
\begin{aligned}
& \lambda^{1 / 2} e^{\alpha a / 2} \cdot \text { Wronskian }\left[J_{0}(z), Y_{0}(z)\right]_{z \min 2 \lambda / 2 e^{\alpha a / 2 / \alpha}} \\
& =\lambda^{1 / 2} e^{\alpha a / 2}\left[J_{0}(z) Y_{0}^{\prime}(z)-J_{0}^{\prime}(z) Y_{0}(z)\right]_{z \rightarrow 2 \lambda 1 / 2 e^{\alpha a / 2 / \alpha}} .
\end{aligned}
$$

By (6.7), for $n$ not an integer, we have 
Wronskian $\left(J_{n}(z), Y_{n}(z)\right)=J_{n}(z) Y_{n}^{\prime}(z)-J_{n}^{\prime}(z) Y_{n}(z)$

$$
\begin{aligned}
& =\frac{1}{\sin n \pi}\left[J_{n}(z) J_{n}^{\prime}(z) \cos n \pi-J_{n}(z) J_{-n}^{\prime}(z)\right. \\
& \left.\quad-J_{n}^{\prime}(z) J_{n}(z) \cos n \pi+J_{n}(z) J_{-n}^{\prime}(z)\right] \\
& =\frac{1}{\sin n \pi}\left[J_{n}^{\prime}(z) J_{-n}(z)-J_{n}(z) J_{-n}^{\prime}(z)\right] .
\end{aligned}
$$

Now it is known that

$$
J_{n}(z) J_{-n}^{\prime}(z)-J_{-n}(z) J_{n}^{\prime}(z)=-2 \sin n \pi / \pi z,
$$

see $[6$, pp. 360,380$]$. Hence $(6.10)$ becomes

$$
\text { Wronskian }\left(J_{n}(z), Y_{n}(z)\right)=\frac{1}{\sin n \pi} \frac{2 \sin n \pi}{\pi z}=\frac{2}{\pi z},
$$

this holding whenever $n$ is not an integer. On taking limits one can easily show that

$$
\text { Wronskian }\left(J_{n}(z), Y_{n}(z)\right)=2 / \pi z
$$

still prevails when $n$ is an integer. Hence (6.8) and (6.9) are equal to

$$
\lambda^{1 / 2} e^{\alpha a / 2} \frac{2}{\pi} \frac{\alpha}{2 \lambda^{1 / 2} e^{\alpha a / 2}}=\frac{\alpha}{\pi} .
$$

On using this in relation ( 0.11 ) of Theorem 1 we find

$$
\begin{aligned}
\int_{C}^{W} & \exp \left(\lambda \int_{0}^{1} x^{2}(t) e^{\alpha t} d t\right) d_{w} x \\
=\frac{\alpha^{1 / 2}}{\pi^{1 / 2} \lambda^{1 / 4} e^{\alpha / 4}} \cdot\left[Y_{0}^{\prime}\left(\frac{2 \lambda^{1 / 2} e^{\alpha / 2}}{\alpha}\right) J_{0}\left(\frac{2 \lambda^{1 / 2}}{\alpha}\right)\right. & \left.-J_{0}^{\prime}\left(\frac{2 \lambda^{1 / 2} e^{\alpha / 2}}{\alpha}\right) Y_{0}\left(\frac{2 \lambda^{1 / 2}}{\alpha}\right)\right]^{-1 / 2},
\end{aligned}
$$

this holding for $\alpha$ real and different from zero, and for $-\infty<\lambda<0$ or $0<\lambda<\lambda_{0}$, where $\lambda_{0}$ is the least characteristic value of the differential equation (6.1) with the boundary conditions $f(0)=f^{\prime}(1)=0$.

7. Example 4, $p(t)=(t+\alpha)^{\beta}, 0<\alpha<\infty, \beta \neq-2$. In this example we let $\beta$ range over all real values other than the value -2 . This value has already been handled in Example 2 given in $\$ 5$. With this choice of $p(t)$, the differential equation $(0.8)$ becomes

$$
f^{\prime \prime}(t)+\lambda(t+\alpha)^{\beta} f(t)=0 \text {. }
$$


We take as a trial solution of (7.1) the series

$$
f(t)=\sum_{n=0}^{\infty} a_{n}(t+\alpha)^{(\beta+2) n+1} .
$$

Then formally

$$
\begin{aligned}
f^{\prime \prime}(t) & =\sum_{n=1}^{\infty} a_{n} n(\beta+2)[n(\beta+2)+1](t+\alpha)^{(\beta+2) n-1} \\
& =-\lambda \sum_{n=0}^{\infty} a_{n}(t+\alpha)^{(\beta+2) n+\beta+1} \\
& =-\lambda \sum_{n=1}^{\infty} a_{n-1}(t+\alpha)^{(\beta+2) n-1} .
\end{aligned}
$$

Thus we find the recurrence relations

$$
n(\beta+2)[n(\beta+2)+1] a_{n}=-\lambda a_{n-1}
$$

or

$$
\begin{aligned}
a_{n} & =-\frac{\lambda a_{n-1}}{(\beta+2)^{2}(n+1 /(\beta+2)) n} \\
& =\frac{(-1)^{n} a_{0} \lambda^{n}}{(\beta+2)^{2 n}(n+1 /(\beta+2))(n-1+1 /(\beta+2)) \cdots(1+1 /(\beta+2)) n !}
\end{aligned}
$$

This suggests that we take

$$
\begin{aligned}
& f^{(1)}(t)=(t+\alpha)^{1 / 2} J_{1 /(\beta+2)}\left[\frac{2(t+\alpha)^{\beta / 2+1}}{\beta+2} \lambda^{1 / 2}\right], \\
& f^{(2)}(t)=(t+\alpha)^{1 / 2} Y_{1 /(\beta+2)}\left[\frac{2(t+\alpha)^{\beta / 2+1} \lambda^{1 / 2}}{\beta+2}\right],
\end{aligned}
$$

and it can be shown easily that these represent two linearly independent solutions of (7.1), for $\lambda$ real and different from zero. As an example we shall show that $f^{(1)}(t)$ satisfies the differential equation (7.1).

For convenience in writing we set

$$
z=\frac{2(t+\alpha)^{\beta / 2+1}}{\beta+2} \lambda^{1 / 2} .
$$

With this notation we find from (7.6)

(7.9) $f^{(1) \prime}(t)=(1 / 2)(t+\alpha)^{-1 / 2} J_{1 /(\beta+2)}(z)+\lambda^{1 / 2}(t+\alpha)^{\beta / 2+1 / 2} J_{1 /(\beta+2)}^{\prime}(z)$ and 


$$
\begin{aligned}
f^{(1) \prime \prime}(t)= & -(1 / 4)(t+\alpha)^{-3 / 2} J_{1 /(\beta+2)}(z) \\
& +\lambda^{1 / 2}(t+\alpha)^{\beta / 2-1 / 2}(\beta / 2+1) J_{1 /(\beta+2)}^{\prime}(z) \\
& +\lambda(t+\alpha)^{\beta+1 / 2} J_{1 /(\beta+2)}^{\prime \prime}(z)
\end{aligned}
$$

Hence

$$
\begin{aligned}
f^{(1)^{\prime \prime}(t)+\lambda(t+\alpha)^{\beta} f^{(1)}(t)} & \\
=\lambda(t+\alpha)^{\beta+1 / 2} & {\left[J_{1 /(\beta+2)}^{\prime \prime}(z)+\frac{1}{z} J_{1 /(\beta+2)}^{\prime}(z)\right.} \\
& \left.-\frac{1}{z^{2}(\beta+2)^{2}} J_{1 /(\beta+2)}(z)+J_{1 /(\beta+2)}(z)\right]
\end{aligned}
$$

and this is zero by virtue of Bessel's differential equation. Hence $f^{(1)}(t)$ defined as in (7.6) is a solution of (7.1). Similarly (7.7) is also a solution of (7.1), and it is linearly independent.

Next we calculate

$$
\begin{aligned}
& f^{(2)^{\prime}}(a) f^{(1)}(a)-f^{(1)^{\prime}}(a) f^{(2)}(a) \\
& =\lambda^{1 / 2}(a+\alpha)^{\beta / 2+1}\left[Y_{1 /(\beta+2)}^{\prime}(z) J_{1 /(\beta+2)}(z)-J_{1 /(\beta+2)}^{\prime}(z) Y_{1 /(\beta+2)}(z)\right] .
\end{aligned}
$$

By (6.13) and (7.8) we find that this becomes

$$
\begin{aligned}
& f^{(2)^{\prime}}(a) f^{(1)}(a)-f^{(1)^{\prime}}(a) f^{(2)}(a) \\
& =\lambda^{1 / 2}(a+\alpha)^{\beta / 2+1} \frac{2}{\pi} \frac{\beta+2}{2(t+\alpha)^{\beta / 2+1} \lambda^{1 / 2}}=\frac{\beta+2}{\pi} .
\end{aligned}
$$

Also by (7.9) and the recurrence formula for Bessel functions we find

$$
\begin{aligned}
f^{(1) \prime}(t) & =\lambda^{1 / 2}(t+\alpha)^{\beta / 2+1 / 2}\left[J_{1 /(\beta+2)}^{\prime}(z)+\frac{1}{z(\beta+2)} J_{1 /(\beta+2)}(z)\right] \\
& =\lambda^{1 / 2}(t+\alpha)^{\beta / 2+1 / 2} J_{1 /(\beta+2)-1}(z) .
\end{aligned}
$$

Similarly

$$
f^{(2)^{\prime}}(t)=\lambda^{1 / 2}(t+\alpha)^{\beta / 2+1 / 2} Y_{1 /(\beta+2)-1}(z) .
$$

Hence relation ( 0.11 ) of Theorem 1 yields

$$
\begin{aligned}
\int_{C}^{W} & \exp \left(\lambda \int_{0}^{1}(t+\alpha)^{\beta} x^{2}(t) d t\right) d_{w} x \\
= & \frac{(\beta+2)^{1 / 2}}{\pi^{1 / 2} \lambda^{1 / 4}(1+\alpha)^{(\beta+1) / 4} \alpha^{1 / 4}} \\
& \cdot\left[Y_{1 /(\beta+2)-1}\left(z_{1}\right) J_{1 /(\beta+2)}\left(z_{0}\right)-J_{1 /(\beta+2)-1}\left(z_{1}\right) Y_{1 /(\beta+2)}\left(z_{0}\right)\right]^{-1 / 2},
\end{aligned}
$$


where

$$
z_{0}=2 \frac{\alpha^{\beta / 2+1} \lambda^{1 / 2}}{\beta+2}, \quad z_{1}=2 \frac{(1+\alpha)^{\beta / 2+1} \lambda^{1 / 2}}{\beta+2} .
$$

The relation (7.16) holds for $\beta \neq-2,0<\alpha<\infty$, and $-\infty<\lambda<\lambda_{0}$, $\lambda \neq 0$.

It is perhaps interesting to put $\beta=0$ in (7.16). We obtain

$$
\begin{aligned}
\int_{C}^{W} \exp \left(\lambda \int_{0}^{1} x^{2}(t) d t\right) d_{w} x & =\frac{2^{1 / 2}}{\pi^{1 / 2} \lambda^{1 / 4}(1+\alpha)^{1 / 4} \alpha^{1 / 4}} \\
\cdot\left[Y_{-1 / 2}\left((1+\alpha) \lambda^{1 / 2}\right)\right. & J_{1 / 2}\left(\alpha \lambda^{1 / 2}\right) \\
& \left.-J_{-1 / 2}\left((1+\alpha) \lambda^{1 / 2}\right) Y_{1 / 2}\left(\alpha \lambda^{1 / 2}\right)\right]^{-1 / 2}
\end{aligned}
$$

Now $[6$, pp. 364, 370]

$$
\begin{aligned}
J_{1 / 2}(u) & =(2 / \pi u)^{1 / 2} \sin u, \quad J_{-1 / 2}(u)=(2 / \pi u)^{1 / 2} \cos u, \\
Y_{1 / 2}(u) & =\frac{J_{1 / 2}(u) \cos (\pi / 2)-J_{-1 / 2}(u)}{\sin (\pi / 2)}=-\left(\frac{2}{\pi u}\right)^{1 / 2} \cos u, \\
Y_{-1 / 2}(u) & =\frac{J_{-1 / 2}(u) \cos (-\pi / 2)-J_{1 / 2}(u)}{\sin (-\pi / 2)}=+\left(\frac{2}{\pi u}\right)^{1 / 2} \sin u .
\end{aligned}
$$

Hence (7.17) becomes

$$
\int_{C}^{W} \exp \left(\lambda \int_{0}^{1} x^{2}(t) d t\right) d_{w} x=\frac{2^{1 / 2}}{\pi^{1 / 2} \lambda^{1 / 4}(1+\alpha)^{1 / 4} \alpha^{1 / 4}}
$$

$$
\cdot\left[\frac { 2 } { \pi ( 1 + \alpha ) ^ { 1 / 2 } \lambda ^ { 1 / 2 } \alpha ^ { 1 / 2 } } \left\{\sin \left((1+\alpha) \lambda^{1 / 2}\right) \sin \left(\alpha \lambda^{1 / 2}\right)\right.\right.
$$

$$
\left.\left.+\cos \left((1+\alpha) \lambda^{1 / 2}\right) \cos \left(\alpha \lambda^{1 / 2}\right)\right\}\right]^{-1 / 2}=\frac{1}{\left(\cos \lambda^{1 / 2}\right)^{1 / 2}}
$$

which yields our earlier result.

\section{BIBLIOGRAPHY}

1. N. Wiener, Generalized harmonic analysis, Acta Math. 55 (1930) pp. 117-258, esp. pp. 214-234. (References to the author's earlier work on the integral are given here.)

2. R. H. Cameron and W. T. Martin, Transformations of Wiener integrals under a general class of linear transformations, submitted to Transactions of American Mathematical Society.

3. E. L. Ince, Ordinary differential equations, London, 1927.

4. R. H. Cameron and W. T. Martin, Transformations of Wiener integrals under translations, Ann. of Math. vol. 45 (1944) pp. 386-396. 
5. R. E. A. C. Paley and N. Wiener, Fourier transforms in the complex domain, Amer. Math. Soc. Colloquium Publications, vol. 19, 1934, New York, pp. 151 and 156.

6. E. T. Whittaker and G. N. Watson, $A$ Course of modern analysis, Cambridge, 4th ed., 1927.

The Massachusetts Institute of Technology and

SYRACUSE UNIVERSITY

\section{A GENESIS FOR CESÃRO METHODS}

\section{RALPH PALMER AGNEW}

1. Introduction. The Cesàro methods $C_{r}$, introduced by Cesàro ${ }^{1}$ because of their applicability to Cauchy products of series, constitute the most publicized class of methods of summability.

The regular Nörlund methods ${ }^{2}$ of summability constitute one of the two most publicized general classes of consistent methods of summability. The regular Hurwitz-Silverman-Hausdorff methods constitute the other.

This note proves the following theorem.

THEOREM. The Cesaro methods are the only methods of summability, regular or not, which are both Nörlund methods and Hurwitz-SilvermanHausdorff methods.

Thus if the Cesàro methods had not been previously introduced into mathematical literature, they could be defined and exploited as the unique class of methods of summability enjoying all of the properties of Nörlund methods and all of the properties of Hurwitz-Silverman-Hausdorff methods.

In $\$ 4$, it is shown that the only methods which are both Riesz methods and Hurwitz-Silverman-Hausdorff methods are methods $\Gamma_{r}$ closely related to the methods $C_{r}$.

2. Nörlund methods. Each sequence $p_{0}, p_{1}, \cdots$ of real or complex constants for which $P_{n} \equiv p_{0}+p_{1}+\cdots+p_{n} \neq 0$ for each $n$ defines a Nörlund method of summability by means of which a sequence $s_{0}, s_{1}, \cdots$ is summable to $\sigma$ if $\sigma_{n} \rightarrow \sigma$ where

Presented to the Society, August 14, 1944; received by the editors June 19, 1944.

1 E. Cesàro, Sur la multiplication des series, Bull. Sci. Math. (2) vol. 14 (1890) pp. 114-120.

2 N. E. Nörlund, Sur une application des fonctions permutables, Lunds Universitets Arsskrift (2) vol. 16 (1919). 